\title{
Effective PRCs Positioning for PAPR Reduction Using PRCs in OFDM Systems
}

\author{
S.G. Yew ${ }^{*}$ and C.E. Tan ${ }^{*}$ \\ Faculty of Computer Science and Information Technology, \\ Universiti Malaysia Sarawak \\ 94300 Kota Samarahan, Sarawak, Malaysia. \\ sgyew912@yahoo.com.cn", cetan@fit.unimas.my*
}

\begin{abstract}
In this paper, the effective use of peak reduction carrier (PRC) for PAPR reduction is evaluated. By taking into account the number of PRCs, its position and PRC-to-Data ratio, the recommendation on how to effectively adopt PRC for PAPR reduction is carried out for OFDM systems with sub-carriers from 64 sub-carriers to 8192 sub-carriers. From our simulations, it has been shown that there are effective positions for PRC allocation as well as a balance needs to be strike for transmission efficiency versus PAPR reduction effectiveness evaluated via the PRC-to-Data ratio.
\end{abstract}

Index Terms - Peak Reduction Carriers, Orthogonal Frequency Division Multiplexing (OFDM)

\section{INTRODUCTION}

Orthogonal Frequency Division Multiplexing (OFDM) is an emerging digital multicarrier modulation scheme, which uses a large number of closely-spaced orthogonal sub-carriers for data transmission. In practice, OFDM signals are generated using the Fast Fourier Transform (FFT) technique in order to achieve orthogonality among sub-carriers for high spectral efficiency.

The main disadvantage of multicarrier modulation is its inheritance of high peak-to-average power ratio (PAPR) in the resultant signal. Owing to this unpredictable high PAPR, in order to minimize signal clipping and distortion, a highly linear amplifier is needed otherwise the clipping at the signal peaks would yield a distortion of the transmitted signal as well as out-of-band radiation. The high PAPR of OFDM signal can be reduced in several ways, such as using coding techniques, mapping techniques, peak reduction carriers and etc.

\section{BACKGROUND}

The high PAPR of OFDM signal has attracted many research attentions where it can be reduced in a number of ways. For example, in the block coded amplitude clipping and filtering technique [6], both the block coding and clipping technique are applied. The high peak of the signal is clipped by the amplitude clipper and a frequency domain filtering is used to filter the out-of-band radiation which occurs due to clipping of an oversampled signal.

Selective Mapping (SLM) [4] is a technique where the transmitter multiplies an OFDM data by several phase sequences in parallel to generate different candidate signals and only the candidate signals with the lowest PAPR is selected for transmission. SLM raised the problem where it needs to transmit the side information to the receiver to indicate how the transmitter generates the signals. It also involves high computational complexity due to calculation of large inverse fast Fourier transform (IFFT) and the long processing of phase optimization.

Tone Reservation (TR) [5] is another technique proposed to efficiently reduce the high PAPR of the OFDM signal. In [5], certain amount of OFDM sub-carriers is reserved. The reserved sub-carriers do not carry any data information, but are used for reducing PAPR. This technique restricts the data vector and the peak reduction vector to lie in disjoint frequency subspaces. The TR scheme shows very good PAPR reduction performance when operating with optimized peak reduction tones (PRT) position but it is not easy to find the optimal PRT positions, and the TR scheme degrades with nonoptimal PRT positions. This technique has a similar nature of Peak Reduction Carriers (PRCs) proposed earlier in [3]. PRC can be more effective in minimizing the PAPR if the effective PRC position is known which is the drive of this research.

\section{OFDM SIGNAL AND PAPR MODEL}

For an OFDM signal consists of a total of $N$ sub-carriers that are modulated by using phase shift keying (PSK) or quadrature amplitude modulation (QAM), assume $X_{k}$ is the modulated complex data symbol carried by the $k^{\text {th }}$ sub-carrier, the complex baseband can be expressed as (1) [7]

$$
x(t)=\sum_{k=0}^{N-1} X_{k} \bullet e^{j 2 \Pi f_{k} t}, 0 \leq \mathrm{t} \leq
$$

